\title{
Clinical and Pathological Attributes of Hepatocellular Carcinoma Showing Lack of Restricted Diffusion on Magnetic Resonance Imaging
}

Dearada Wancharoenrung, M.D. ${ }^{1,2}$, Kamonwon Cattapan, M.D. ${ }^{1,3}$, Thitinan Chulroek, M.D. ${ }^{1,4}$, Hamed Kordbacheh ${ }^{1}$, Martina Cecconi, M.D. ${ }^{5}$, Mukesh Harisinghani, M.D. ${ }^{1}$

'Department of Radiology, Massachusetts General Hospital, Harvard Medical School, Boston, MA 02114, United States.

2Department of Diagnostic Radiology, Faculty of Medicine, Vajira Hospital, Nawamindradhiraj University, Dusit, Bangkok 10300, Thailand.

${ }^{3}$ Department of Radiology, Faculty of Medicine, Prince of Songkla University, Hat Yai, Songkhla 90110, Thailand.

${ }^{4}$ Department of Diagnostic Radiology, Faculty of Medicine, Chulalongkorn University and King Chulalongkorn Memorial Hospital, Pathum Wan, Bangkok 10330, Thailand.

${ }^{5}$ Department of Diagnostic Radiology, AOB Azienda Ospedaliera Brotzu, Piazzale Ricchi, Cagliari Sardinia 09134, Italy.

Received 27 April 2020 • Revised 24 November 2020 • Accepted 2 December 2020 • Published online 9 April 2021

\section{Abstract:}

Objective: To correlate non-restricted diffusion magnetic resonance imaging (MRI) patterns of hepatocellular carcinoma (HCC), with histopathology and clinical outcome.

Material and Methods: We retrospectively evaluated pre-treatment MRIs showing non-restricted diffusion HCC lesions ( $\geq 1$-centimeter), excluding lesions with poor quality/non-available diffusion weighted imaging (DWI). Three radiologists evaluated 37 lesions in 27 patients, for: T1-weighted (T1W)/T2-weighted (T2W) characteristics, arterial enhancement, washout on portal venous/delayed phase, capsular enhancement, intralesional fat component and presence of cirrhosis. Histopathological reports were categorized as: well/moderate/poorly differentiated. Kaplan-Meier survival analysis was calculated for clinical outcome.

Results: From a total of 37 lesions, 24 lesions had available pathological grading, which revealed well and moderately differentiated equally (12 lesions each). None of the non-restricted diffusion HCCs were poorly differentiated. Thirty-five of the 37 lesions (94.6\%) showed arterial enhancement with washout; 34 lesions (91.9\%) were T2W hypo-/Isointense, 33

Contact: Dearada Wancharoenrung, M.D.

Department of Diagnostic Radiology, Faculty of Medicine, Vajira Hospital,

Nawamindradhiraj University, Dusit, Bangkok 10300, Thailand.

E-mail: dearada@nmu.ac.th

This is an open access article under the CC BY-NC-ND license

(http://www.jhsmr.org/index.php/jhsmr/about/editorialPolicies\#openAccessPolicy).

J Health Sci Med Res 2021;39(6):447-458 doi: 10.31584/jhsmr.2021802 www.jhsmr.org 
lesions (89.2\%) were T1W iso-Myperintense, 19 lesions (51.4\%) showed capsular enhancement and 8 lesions (21.6\%) had intralesional fat. These findings in the well and moderately differentiated groups were not significantly different ( $p$-value 0.178-1.000). Overall mean-survival was 6.972 years (95\% confidence interval (Cl); 5.3-8.6). The 1-year, overall survival rate was $83.6 \%$ and for 3 -years was $67.9 \%$. Mean survival of well and moderately differentiated groups were 6.88 and 7.23 years $(95 \% \mathrm{Cl} 5.7-8.0$ and $4.4-10.1)$, respectively ( $\mathrm{p}$-value=0.319).

Conclusion: DWI may help to predict histological grading of HCC and clinical outcome. We found that non-restricted diffusion HCCs were histologically well or moderately differentiated, with no significant difference of imaging findings and survival rates between the two groups. No poorly differentiated lesions were seen in our non-restricted HCC cohort.

Keywords: diffuse weighted imaging, hepatocellular carcinoma, histological grading, liver, non-restricted diffusion

\section{Introduction}

Hepatocellular carcinoma $(\mathrm{HCC})$ is the most common, primary malignancy of the liver. It is the fifth most common cancer in men and seventh among women; with over half a million new cases diagnosed annually worldwide. ${ }^{1,2} \mathrm{HCC}$ has been the leading indication for liver transplantation in the United States since 2015 . $^{3}$

Unlike most other cancers, HCC can be conclusively diagnosed on imaging studies; without need of tissue sampling confirmation. ${ }^{4}$ Imaging criteria, with high specificity, for 1-centimeter (cm) HCC or larger has been developed through Liver Imaging Reporting and Data System (LIRADS), the Organ Procurement and Transplantation Network classification, and the American Association for the Study of Liver Diseases (AASLD) guidelines. ${ }^{5-7}$ These guidelines include; late arterial enhancement followed by washout patterns relative to the liver parenchyma, during the venous or delayed phases (3-5 minutes post injection).

For masses that cannot be categorized as definite HCC, based on enhancement patterns, additional features of HCC; including, capsular enhancement, T2-weighted (T2W) hyperintensity, restricted diffusion and intralesional fat content, have also shown to be specific for $\mathrm{HCC}^{4}$
Highly cellular tissue, such as tumors, restricts the apparent diffusion of water, due to tortuosity of the extracellular space and the higher density of hydrophobic cellular membranes. ${ }^{8}$ Diffusion weighted imaging (DWI) can be used to detect and characterize liver lesions with better results, when compared with $\mathrm{T} 2 \mathrm{~W}$ imaging. ${ }^{9}$ The presence of diffusion restriction favors the diagnosis of malignancy, and also helps differentiate HCC from dysplastic nodules in patients with cirrhosis. ${ }^{10}$ In addition, DWI can be used as an alternative imaging procedure in patients who cannot receive gadolinium-based contrast agents. ${ }^{8}$ While most HCCs show restricted diffusion, some may not demonstrate the usual restriction pattern. Nasu et al. reported that $91.2 \%$ of HCC showed a hyperintense signal compared to the surrounding hepatic parenchyma on DWI sequences. ${ }^{11}$ Park et al. ${ }^{12}$ also showed that DWI can detect HCC, with a sensitivity of $75.8 \%$ and specificity of $81.6 \%$.

To our knowledge, no study has directly evaluated the non-restricted diffusion type of HCC, and correlated them with pathological grading and clinical outcome. Hence, the purpose of this study was to correlate the non-restricted diffusion magnetic resonance imaging (MRI) pattern of HCC, with histopathology and clinical outcome. 


\section{Material and Methods}

Institutional Review board approval was obtained for this retrospective study, and requirement for informed consent was waived.

We found 1,016 MRI studies of patients with clinically suspected HCC, performed between January, 2008 to December, 2017; as identified by searching our radiology database, which queried radiologic image data from the diagnostic Picture Archiving and Communication System (PACS) workstations (AGFA Impax; AGFA Technical Imaging Systems, Ridgefield Park, NJ).

After defining patients with a confirmed diagnosis of HCC, either by pathologic confirmation (core needle biopsy or surgical resection) or imaging criteria (based on 2016 AASLD imaging criteria for diagnosis of HCC which included arterial phase enhancement greater than background liver and contrast washout below background liver on portal venous or delayed phase images), we found 679 cases. Then we selected HCC lesions that have pre-treatment MRI available in our PACS, either done in our institution or from other institutions, which resulted in 601 cases.

We applied the exclusion criteria of HCC as: smaller than $1 \mathrm{~cm}$ in diameter (8 cases), no DWI sequences available (26 cases), poor DWI quality of the HCC lesion (3 cases) and those showing restricted diffusion in DWI (564 cases).

In cases with multiple lesions, all lesions were evaluated separately. The final patient cohort consisted of 37 lesions, in 27 patients (Figure 1).

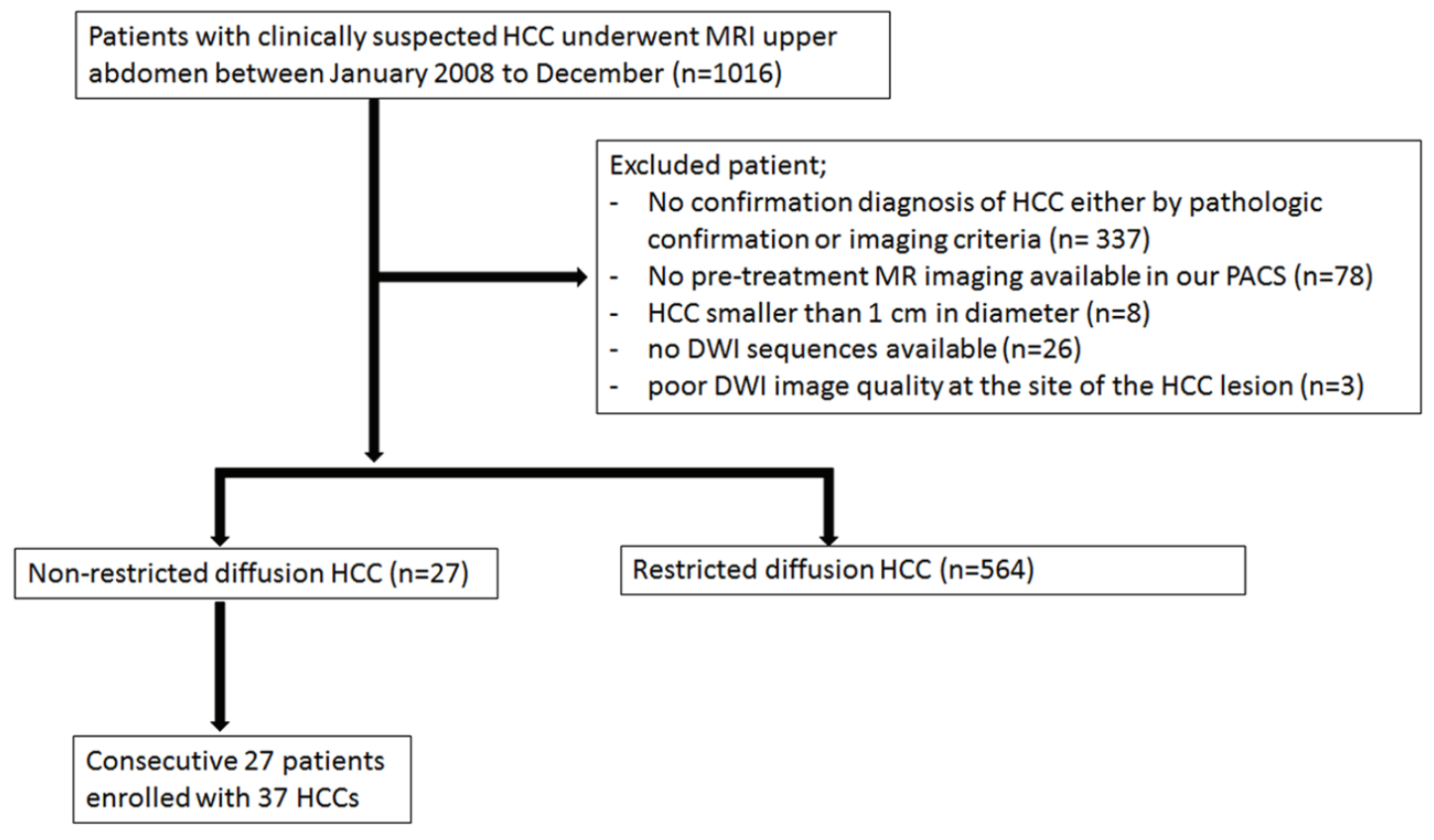

$\mathrm{HCC}=$ hepatocellular carcinoma, MRI=magnetic resonance imaging, PACS=picture archiving and communication system, DWI=diffuse weighted image

Figure 1 Flowchart of the study population 
Although, the included cohort comprised of patients scanned locally at our institution $(n=22)$ as well as at other institutions $(n=5)$, care was taken to ensure the MRI protocol was homogenous, and comprised of all the essential sequences. The studies were performed mainly on 1.5-Tesla scanners $(n=21)$, with a subset performed on 3-Tesla scanners $(n=6)$, using body phased-array coils.

The minimum sequences included: T1-weighted (T1W) dual-echo (in-phase and opposed-phase) and T1W images with fat-saturated gradient-recalled echo, T2W single-shot or multi-shot sequences, dynamic contrastenhanced multiphasic T1W and DWI sequence. The DWI sequence comprised of at least two b-values; one in low range [0-100 seconds per square millimeter $\left(\mathrm{sec} / \mathrm{mm}^{2}\right)$ ] and one in intermediate to high range (400-800 sec/ $\mathrm{mm}^{2}$ ). An apparent diffusion coefficient (ADC) map was also generated. T2W images were acquired before or after contrast agent administration.

The HCC lesions were read by 3 radiologists, having 3,4 , and 8 years of experience in general diagnostic radiology in separate sessions. They were blinded to patient identity, clinical history, other imaging examinations as well as histopathological results.

The HCC lesions that showed higher signal intensity, compared to liver background on low b-value (0-100 sec/ $\mathrm{mm}^{2}$ ), remained hyperintense on intermediate and high b-values (>400 sec/ $/ \mathrm{mm}^{2}$ ), and showed lower or equal signal intensity compared to liver background on ADC map were interpreted as showing restricted diffusion. Lesions that did not meet these criteria were labelled as showing no restricted diffusion.

The lesions with no restricted diffusion pattern were evaluated, by said 3 radiologists, for the presence of background liver cirrhosis, number of HCC lesion and tumor location (right/left/caudate lobe). Lesion size was measured as the largest outer-edge-to-outer-edge dimension, including capsule on the axial plane. The radiologists also interpreted the presence of: arterial enhancement, washout on portovenous or delayed phase, capsular enhancement, signal intensity on T1W and T2W images (compared with the liver parenchyma) and intralesional fat component. Intralesional fat HCC was defined as: hyperintense on T1W images with drop signal intensity on opposed phase of chemical shift images.

Lesions were categorized as well, moderate, and poorly differentiate of HCC; based on available histopathological results.

The continuous variables were computed using the paired Student t-test and analysis of variance. The Chisquare test was used for comparing categoric variables. Survival analysis with overall survival curves were estimated using the Kaplan-Meier method, and compared using the log-rank test. Two-sided $\mathrm{p}$-values $<0.05$ were considered to indicate statistical significance. Medical statistical software package (MedCalc version 18.2.1; Medcalc Software, Ostend, Blegium) was used to calculate statistics.

\section{Results}

From the inclusion and exclusion criteria, as described above, 37 lesions in 27 patients were finally included in the study; 19 were men and 8 were women (mean age of $62 \pm 8.55$ years, age range: $44-78$ years). Twenty-six out of 27 patients (96.3\%) had liver cirrhosis, which was diagnosed by pathological report or MR imaging features. One patient without liver cirrhosis was a hepatitis B viral carrier. Twenty patients (74.1\%) had a single lesion, while 7 patients (25.9\%) had multiple lesions (2-3 lesions) (Table 1).

Twenty-four HCCs (64.9\%) were located in the right hepatic lobe, left hepatic lobe in 12 lesions (32.4\%), and caudate lobe in 1 lesion (2.7\%). The sizes of HCCs ranged from 1.1 to $5.7 \mathrm{~cm}$ in maximal dimensions, with a mean size of $1.9 \mathrm{~cm}$ (Table 2). 
Table 1 Patient's characteristics $(n=27)$

\begin{tabular}{ll}
\hline Patient's characteristic $(\mathbf{n = 2 7 )}$ & Value \\
\hline Mean age (years) & $62 \pm 8.55(44-78)$ \\
Gender & $19(70.4 \%)$ \\
Men & $8(29.6 \%)$ \\
Woman & $26(96.3 \%)$ \\
Liver cirrhosis & \\
Number of HCCs & $20(74.1 \%)$ \\
Single & $7(25.9 \%)$ \\
Multiple (2-3 lesions) & \\
Treatment & $7(25.9 \%)$ \\
Liver transplantation & $7(25.9 \%)$ \\
RFA & $4(14.8 \%)$ \\
Partial hepatectomy & $3(11.1 \%)$ \\
TACE & $3(11.1 \%)$ \\
During decision & $2(7.4 \%)$ \\
Combined RFA and TACE & $1(3.7 \%)$ \\
Refuse treatment &
\end{tabular}

HCC=Hepatocellular carcinoma, RFA=radiofrequency ablation,

TACE=transarterial chemoembolization
The HCC lesions were interpreted as those showing arterial phase enhancement with washout on portovenous or delayed images, for 35 lesions out of 37 lesions (94.6\%). The other 2 lesions (5.4\%) showed arterial phase enhancement, without evidence of washout on delayed images and were pathologically proven as HCC. Capsular enhancement was found in 19 cases (51.4\%) (Table 2).

Based on signal intensity, the lesions were classified as: T2W hyperintense for 3 lesions (8.1\%) and T2W iso- to hypointense for 34 lesions (91.9\%). T1W signal intensity was classified as: hyperintense for 20 lesions (54.1\%), isointense for 13 lesions (35.1\%) and hypointense for 4 lesions (10.8\%). Intralesional fat was found in 8 out of 37 lesions (21.6\%) (Table 2). Examples of imaging interpretations are shown in Figures 2 and 3.

Table 2 Lesions characteristic

\begin{tabular}{|c|c|c|c|c|}
\hline Lesions characteristic & $\begin{array}{l}\text { All HCC lesions } \\
(n=37)\end{array}$ & $\begin{array}{l}\text { Well differentiated HCC } \\
(n=12)\end{array}$ & $\begin{array}{l}\text { Moderately differentiated HCC } \\
(n=12)\end{array}$ & $p$-value \\
\hline Location of tumor & & & & 0.1779 \\
\hline Right lobe & $24(64.9)$ & $10(83.3)$ & $7(58.3)$ & \\
\hline Left lobe & $12(32.4)$ & $2(16.7)$ & $5(41.7)$ & \\
\hline Caudate lobe & $1(2.7)$ & 0 & 0 & \\
\hline $\begin{array}{l}\text { Mean size of HCCs } \\
\text { Lesions characteristic }\end{array}$ & $1.9 \mathrm{~cm}: 1.1-5.7 \mathrm{~cm}$ & $2.1 \mathrm{~cm}: 1.1-3.9 \mathrm{~cm}$ & $2.2 \mathrm{~cm}: 1.2-5.7 \mathrm{~cm}$ & 0.5004 \\
\hline Arterial enhancement & $37(100.0)$ & $12(100.0)$ & $12(100.0)$ & 1.000 \\
\hline Washout & $35(94.6)$ & $12(100.0)$ & $11(91.7)$ & 0.307 \\
\hline Capsular enhancement & $19(51.4)$ & 7 (58.3) & 7 (58.3) & 1.000 \\
\hline T2W hyperintensity & $3(8.1)$ & 0 & $1(8.3)$ & 0.5276 \\
\hline T1W hypointensity & $4(10.8)$ & 0 & 0 & - \\
\hline Intralesional fat & $8(21.6)$ & $5(41.7)$ & $2(16.7)$ & 0.1779 \\
\hline
\end{tabular}

Note-Data in parentheses are percentages. Dash (-) indicates p-value cannot be calculated.

HCC=hepatocellular carcinoma, $\mathrm{T} 2 \mathrm{~W}=\mathrm{T} 2$-weighted, $\mathrm{T} 1 \mathrm{~W}=\mathrm{T} 1$-weighted 

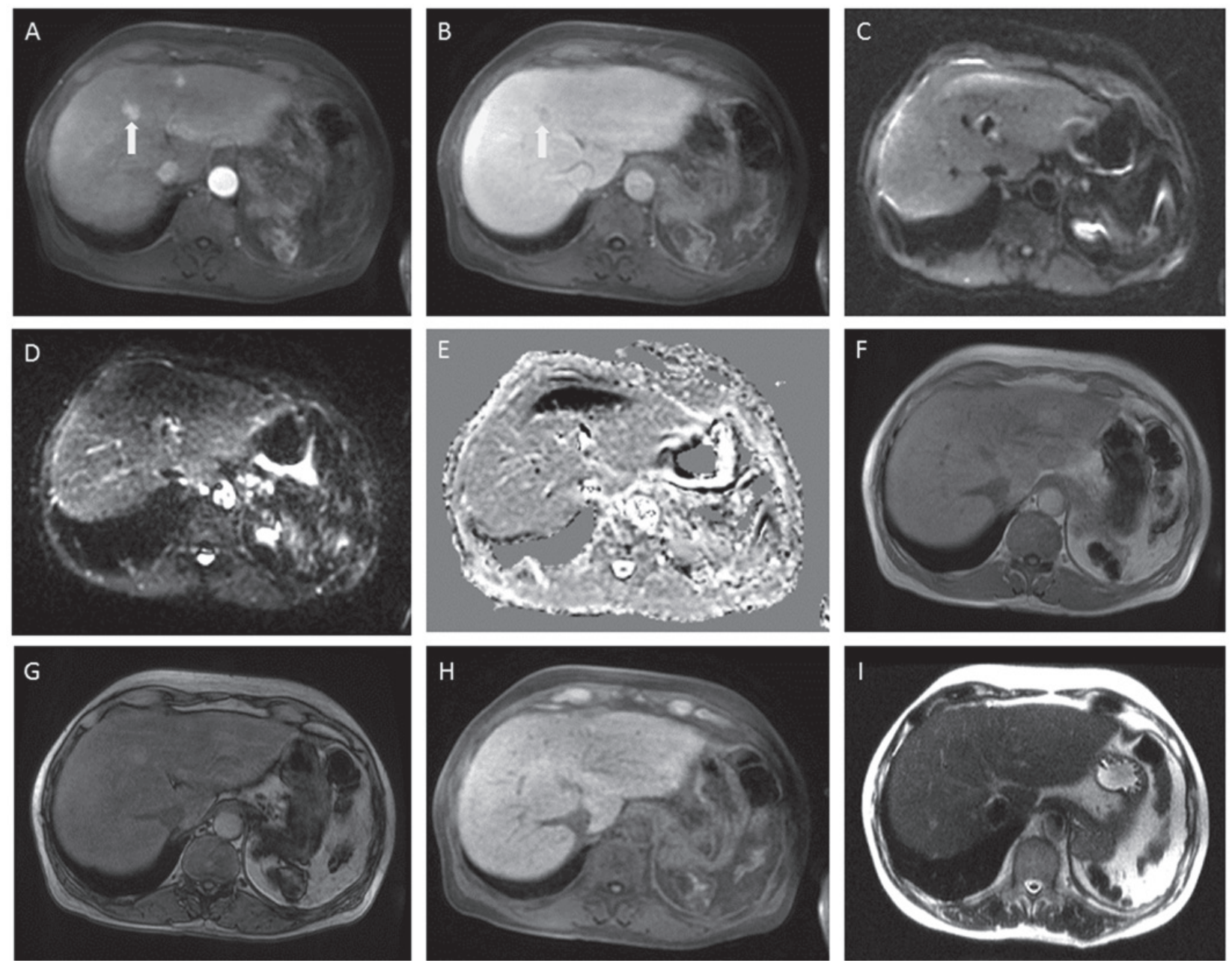

Figure 2 A 64-year-old man, with cirrhosis containing a biopsy-proven moderately differentiated hepatocellular carcinoma (arrows).

Axial arterial phase image (A) and delayed magnetic resonance images obtained 180 seconds after administration of gadolinium (B) shows hepatocellular carcinoma with arterial enhancement, washout on delayed phase and faint capsular enhancement.

Single-shot echo-planar diffuse weighted images at b-value=100 sec/ $/ \mathrm{mm}^{2}$ (C) and b-value $=600 \mathrm{sec} / \mathrm{mm} \mathrm{m}^{2}$ (D) and apparent diffusion coefficient mapping (E) shows isosignal intensity. All observers diagnosed hepatocellular carcinoma, with no restricted diffusion.

In-phase spoiled gradient-echo magnetic resonance image (F) shows isosignal intensity of the hepatocellular carcinoma without drop in signal intensity of the tumor in corresponding opposed-phase spoiled gradientecho magnetic resonance image $(\mathrm{G})$ and interpreted as no intra-lesional fat.

Fast spoiled gradient-echo T1-weighted image $(\mathrm{H})$ and T2-weighted single shot fast spin-echo image (I) shows isosignal intensity of the hepatocellular carcinoma. 

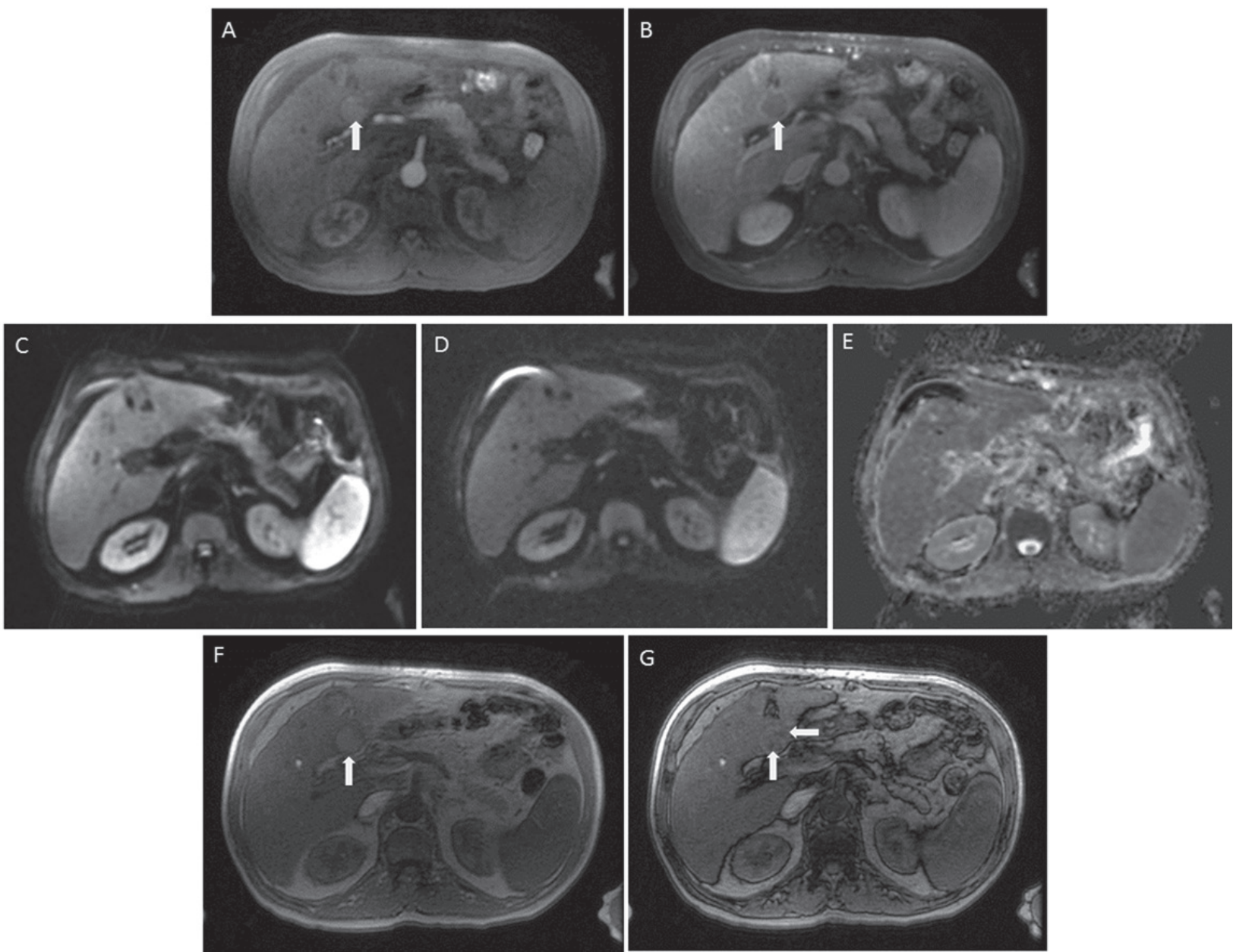

Figure 3 A 67-year-old man, with cirrhosis containing a biopsy-proven moderately differentiated hepatocellular carcinoma at hepatic segment IVB (arrows).

Axial arterial phase image (A) and delayed magnetic resonance, image obtained 180 seconds after administration of gadolinium (B) shows hepatocellular carcinoma with arterial enhancement, washout on delayed phase and capsular enhancement.

Single-shot echo-planar diffuse weighted images at b-value $=100 \mathrm{sec} / \mathrm{mm}^{2}$ (C) and b-value $=800 \mathrm{sec} / \mathrm{mm}^{2}$ (D) and apparent diffusion coefficient mapping (E) shows isosignal intensity. All observers diagnosed hepatocellular carcinoma, with no restricted diffusion.

In-phase spoiled gradient-echo magnetic resonance image (F) shows well-defined hyperintensity mass; which reveals some drop in signal intensity of the tumor in corresponding opposed-phase spoiled gradientecho magnetic resonance image $(G)$ interpreted as containing some intralesional fat. 
Tissue diagnosis of HCC was conducted in 27 lesions out of 19 cases; 12 lesions were well differentiated, 12 lesions were moderately differentiated, and 3 lesions did not mention the type of HCC. No poorly differentiated were found from non-restricted HCC in this study (Table 3). The location of tumors, size, and lesion characteristic of well differentiate and moderately differentiated of these 24 lesions are shown in Table 2. Chi-square test was applied to determine whether there was significant difference between well and moderately differentiated groups, and also shown in the same table. The results revealed no statistically significant difference of findings between these two groups, with a p-value of $0.1779-1.000$ (Table 2).

Table 3 Pathological report

\begin{tabular}{lll}
\hline Pathological report & lesions & Cases \\
\hline Available & 27 & 19 \\
Well differentiated & 12 & 8 \\
Moderately differentiated & 12 & 8 \\
Poorly differentiated & 0 & 0 \\
Not mentioned type & 3 & 3 \\
Not available & 10 & 8 \\
\hline Total & 37 & 27 \\
\hline
\end{tabular}

None of the patients had metastasis at the time of diagnosis. Patients were treated by liver transplantation ( 7 cases), radiofrequency ablation (RFA) (7 cases), transarterial chemoembolization (TACE) (3 cases), combined RFA and TACE (2 cases) and partial hepatectomy (4 cases). One patient refused treatment and three patients were in decision treatment planning at the time of data collection (Table 1). Average follow-up of all patients in this study was 3.015 years. Five patients had recurrent tumors at 7 months, 2 years, 2 years, 4 years, and 5 years. Seven patients died. Kaplan-Meier survival analysis of all non-restricted diffusion HCCs was calculated (Figure 4). Both median overall survival time and disease-free survival time were not reached. Overall mean survival time of 27 patients was 6.972 years from the date of diagnosis, with a 95\% confident interval $(\mathrm{Cl})$ of 5.327 to 8.617. The overall 1-year survival rate was $83.6 \%$ (standard error $=0.0753$ ) and after 3 years was $67.9 \%$ (standard error=0.103).

Kaplan-Meier survival analysis of well and moderately differentiate groups was also calculated (Figure 5). From the both median overall survival time and diseasefree survival time were not reached. The mean survival rate of well and moderately differentiated were 6.876 years (95\% $\mathrm{Cl}=5.715$ to 8.036$)$ and 7.233 years $(95 \% \mathrm{Cl}=5.970$ to 9.626$)$, respectively. There was no statistically significant difference between these two groups ( $p-$ value=0.3192).

The 3-year survival rate of the well differentiated group was $85.7 \%$ (standard error=0.132). The 3-year survival rate of moderately differentiated group and 1-year survival rate of both groups could not be calculated.

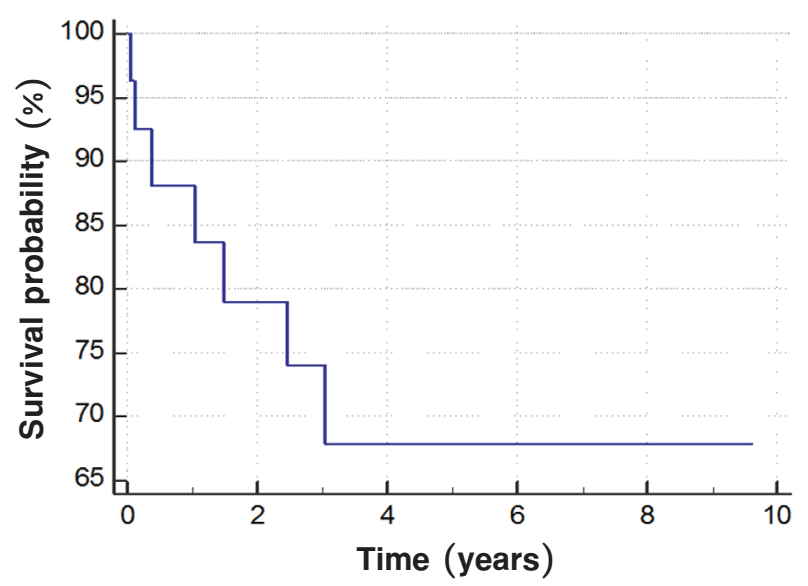

Figure 4 Kaplan-Meier survival analysis of all non-restricted diffusion hepatocellular carcinomas This figure was made by using Medical statistical software package (MedCalc version 18.2.1; Medcalc Software, Ostend, Blegium) 


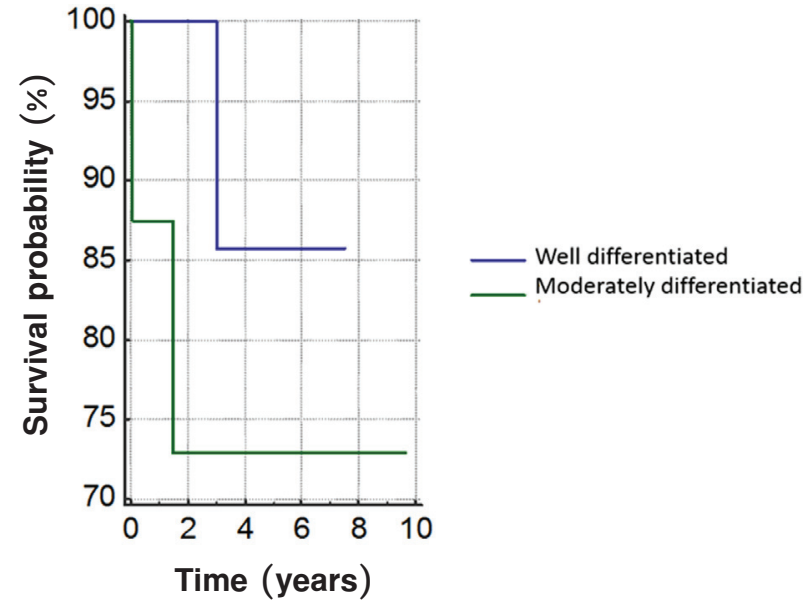

Figure 5 Kaplan-Meier survival analysis of well and moderately differentiated non-restricted diffusion hepatocellular carcinomas

This figure was made by using Medical statistical software package (MedCalc version 18.2.1; Medcalc Software, Ostend, Blegium)

\section{Discussion}

The purpose of this study was to correlate nonrestricted diffusion MRI patterns of HCC with histopathology and clinical outcome. In our study, we found that nonrestricted diffusion HCCs showed well or moderate differentiation (50.0\% each) on histopathology, with none showing poor differentiation. These results were similar to the study of Muhi et al., who reported that more than $90.0 \%$ of moderately and poorly differentiated HCC show restricted diffusion, while approximately $50.0 \%$ of well differentiated HCC and all dysplastic nodules don't. ${ }^{13}$ Many similar studies reported that poorly differentiated HCCs showed significantly lower ADC values than well and moderately differentiated HCCs. ${ }^{14-19}$ Some studies also reported that HCC tended to show a higher signal intensity on DWI as the histological grade rises. ${ }^{11,19,20}$ These could be explained by some of these well and moderately differentiated HCCs not having enough cellular density to restrict water molecule movement, and have minimal difference in cellularity from the background liver parenchyma. ${ }^{21}$

Gluskin et al. suggested that surrounding liver cirrhosis can lead to a false negative DWI of HCC's. ${ }^{22}$ Cirrhotic liver causes restricted diffusion of water molecules as compared to normal parenchyma, which makes it difficult to identify HCC in severely cirrhotic livers compared with noncirrhotic or mildly cirrhotic livers. ${ }^{23,24}$

HCCs are often hyperintense on T2W images. ${ }^{25}$ However, in our study we found that about $92.0 \%$ of non-restricted HCCs were iso-to hypointense T2W. As correlated with the pathological grading, these HCCs were well and moderately differentiation. Some studies have reported that HCC with T2 signal hyperintensity suggested advanced tumor grade. In contrast, some well-differentiated along with some small, moderately differentiated HCCs had signal characteristics similar to cirrhotic nodules and dysplastic nodules, which were isointense or hypointense on T2-weighted images of the liver. ${ }^{26-31}$ Moreover, some HCCs with mild T2 hyperintensity may be obscured by parenchymal fibrosis, and appear to be isointense on $\mathrm{T}_{2} \mathrm{~W}^{32}$ Some studies have also reported that lesions containing iron were often seen as hypointense T2W. As HCC progresses tumor cells lose the ability to concentrate iron and become T2W hypersignal in intensity. ${ }^{33}$

Both HCCs and nonmalignant hepatic nodules have variable and overlapping T1W signal intensity. ${ }^{34}$ We found that non-restricted HCC mostly had hypersignal intensity on T1W, which was correlated with the studies showing that T1-hyperintense HCC nodules tended to have a lower tumor grade. ${ }^{31,35}$ There were also many other factors causing hypersignal intensity of $\mathrm{HCC}$ on T1W images, including hemorrhage, intratumoral fat component and the copper/ zinc content of surrounding liver parenchyma. ${ }^{31}$

It was not surprising that the non-restricted HCCs in our study had an average size of $1.9 \mathrm{~cm}$, which was revealed to be of a low histological grade. In contrast, 
larger HCCs tended to have a higher histologic grade, more aggressive biologic behavior as well as a higher frequency of vascular invasion and metastasis. ${ }^{36}$

We found $21.6 \%$ of HCCs had an intralesional fat component, which was similar to the study of Kutami et al., who reported that intratumoral fat could be seen in $19.6 \%$ via standard light microscopy and up to $10.0 \%$ by MRIs. ${ }^{37}$ Findings of intralesional fat in cirrhotic livers is fairly specific, which is an ancillary feature favoring HCC; according to 2017 LI-RADS, but an insensitive finding for HCC. ${ }^{38}$

We found that the 1-year survival rate was $83.6 \%$ and 3-year survival rate was $67.9 \%$, which were similar to other studies. Altekruse et al., reported the 1-year survival rate of treated HCC in 2003-2004 was $83.0 \%{ }^{39}$ Zhang et al., reported 1-year, 3-year, and 5-year survival rate of HCC surveillance patients were $65.9 \%, 52.6 \%, 46.4 \%$, respectively. ${ }^{40}$

Our study was limited by the small number of lesions, which showed an atypical pattern lacking restricted diffusion. Although, we tried to collect all the cases showing no restricted diffusion, only 37 lesions were included in this study, and only 24 lesions had histological proof of HCC; owing to included lesions with tissue diagnosis before treatment. Furthermore, some cases did not perform high b-valued DWI, therefore the sensitivity to detected $\mathrm{HCC}$ with restricted diffusion in our study was decreased. However, to our knowledge, this was the largest population analysis of non-restricted diffusion HCC in the literature.

The survival analysis was also limited, due to the median overall survival rate and disease-free survival rate not being reached during this 10-year period of study.

\section{Conclusion}

DWI may help to predict histological grading of HCC and clinical outcome. We found that non-restricted diffusion HCCs were histologically well- or moderately differentiated, with no significant difference of imaging findings and survival rates between these two groups. No poorly differentiated lesions were seen in our non-restricted HCC cohort.

\section{Conflict of interest}

The authors declare that they have no conflict of interest.

\section{Reference}

1. Mittal S, El-Serag HB. Epidemiology of HCC: consider the population. J Clin Gastroenterol 2013;47:S2-6.

2. Ghouri YA, Mian I, Rowe JH. Review of hepatocellular carcinoma: Epidemiology, etiology, and carcinogenesis. J Carcinog [serial on the Internet]. 2017 May [cited 2018 Feb 5];16. Available from: https://www.ncbi.nlm.nih.gov/pmc/articles/PMC5490340/

3. Yang JD, Larson JJ, Watt KD, Allen AM, Wiesner RH, Gores GJ, et al. Hepatocellular carcinoma is the most common indication for liver transplantation and placement on the waitlist in the United States. Clin Gastroenterol Hepatol 2017; 15:767.

4. Willatt J, Ruma JA, Azar SF, Dasika NL, Syed F. Imaging of hepatocellular carcinoma and image guided therapies - how we do it. Cancer Imaging 2017;17:9.

5. Elsayes KM, Hooker JC, Agrons MM, Kielar AZ, Tang A, Fowler KJ, et al. 2017 version of LI-RADS for CT and MR imaging: an update. RadioGraphics 2017;37:1994-2017.

6. Wald C, Russo MW, Heimbach JK, Hussain HK, Pomfret EA, Bruix J. New OPTN/NNOS Policy for liver transplant allocation: standardization of liver imaging, diagnosis, classification, and reporting of hepatocellular carcinoma. Radiology 2013;266: 376-82

7. Heimbach JK, Kulik LM, Finn RS, Sirlin CB, Abecassis MM, Roberts LR, et al. AASLD guidelines for the treatment of hepatocellular carcinoma. Hepatol Baltim Md 2018;67:358-80.

8. Taouli B, Koh DM. Diffusion-weighted MR Imaging of the Liver. Radiology 2009;254:47-66.

9. Hicks RM, Yee J, Ohliger MA, Weinstein S, Kao J, Ikram NS, et al. Comparison of diffusion-weighted imaging and T2weighted single shot fast spin-echo: Implications for LI-RADS characterization of hepatocellular carcinoma. Magn Reson Imaging 2016;34:915-21. 
10. Xu PJ, Yan FH, Wang JH, Shan Y, Ji Y, Chen CZ. Contribution of diffusion-weighted magnetic resonance imaging in the characterization of hepatocellular carcinomas and dysplastic nodules in cirrhotic liver. J Comput Assist Tomogr 2010;34: 506-12.

11. Nasu K, Kuroki $Y$, Tsukamoto $T$, Nakajima H, Mori K, Minami M. Diffusion-weighted imaging of surgically resected hepatocellular carcinoma: imaging characteristics and relationship among signal intensity, apparent diffusion coefficient, and histopathologic grade. Am J Roentgenol 2009;193:438-44.

12. Park MS, Kim S, Patel J, Hajdu CH, G. Do RK, Mannelli L, et al. Hepatocellular carcinoma: detection with diffusionweighted versus contrast-enhanced magnetic resonance imaging in pretransplant patients. Hepatology 2012;56:140-8.

13. Muhi A, Ichikawa T, Motosugi U, Sano K, Matsuda M, Kitamura $T$, et al. High-b-value diffusion-weighted MR imaging of hepatocellular lesions: estimation of grade of malignancy of hepatocellular carcinoma. J Magn Reson Imaging 2009;30:1005-11.

14. Nishie A, Tajima T, Asayama Y, Ishigami K, Kakihara D, Nakayama $T$, et al. Diagnostic performance of apparent diffusion coefficient for predicting histological grade of hepatocellular carcinoma. Eur J Radiol 2011;80:e29-33.

15. Heo SH, Jeong YY, Shin SS, Kim JW, Lim HS, Lee JH, et al. Apparent diffusion coefficient value of diffusion-weighted imaging for hepatocellular carcinoma: correlation with the histologic differentiation and the expression of vascular endothelial growth factor. Korean J Radiol 2010;11:295-303.

16. Nakanishi M, Chuma M, Hige S, Omatsu T, Yokoo H, Nakanishi $\mathrm{K}$, et al. Relationship between diffusion-weighted magnetic resonance imaging and histological tumor grading of hepatocellular carcinoma. Ann Surg Oncol 2012;19:1302-9.

17. Tang $Y$, Wang $H, M a L$, Zhang $X, Y u G$, Li J, et al. Diffusionweighted imaging of hepatocellular carcinomas: a retrospective analysis of correlation between apparent diffusion coefficients and histological grade. Abdom Radiol N Y 2016; 41:1539-45.

18. Shenoy-Bhangle A, Baliyan V, Kordbacheh H, Guimaraes AR, Kambadakone A. Diffusion weighted magnetic resonance imaging of liver: principles, clinical applications and recent updates. World J Hepatol 2017;9:1081-91.

19. Guo W, Zhao S, Yang Y, Shao G. Histological grade of hepatocellular carcinoma predicted by quantitative diffusionweighted imaging. Int J Clin Exp Med 2015;8:4164-9.

20. Saito K, Moriyasu F, Sugimoto K, Nishio R, Saguchi T, Akata S, et al. Histological grade of differentiation of hepatocellular carcinoma: comparison of the efficacy of diffusion-weighted MRI with T2-weighted imaging and angiography-assisted CT. J Med Imaging Radiat Oncol 2012;56:261-9.

21. Kim YK, Kim CS, Han YM, Lee YH. Detection of liver malignancy with gadoxetic acid-enhanced MRI: Is addition of diffusionweighted MRI beneficial. Clin Radiol 2011;66:489-96.

22. Gluskin JS, Chegai F, Monti S, Squillaci E, Mannelli L. Hepatocellular Carcinoma and Diffusion-Weighted MRI: Detection and Evaluation of Treatment Response. J Cancer 2016;7:1565-70.

23. Taouli B, Tolia AJ, Losada M, Babb JS, Chan ES, Bannan MA, et al. Diffusion-weighted MRI for quantification of liver fibrosis: preliminary experience. AJR Am J Roentgenol 2007; 189:799-806.

24. Luciani A, Vignaud A, Cavet M, Tran Van Nhieu J, Mallat A, Ruel L, et al. Liver cirrhosis: intravoxel incoherent motion MR imaging—pilot study. Radiology 2008;249:891-9.

25. Gore RM, Levine MS. Textbook of Gastrointestinal Radiology. $4^{\text {th }}$ ed. Philadelphia: Saunders/Elsevier; 2014.

26. Kadoya M, Matsui O, Takashima T, Nonomura A. Hepatocellular carcinoma: correlation of MR imaging and histopathologic findings. Radiology 1992;183:819-25.

27. Choi JY, Lee JM, Sirlin CB. CT and MR imaging diagnosis and staging of hepatocellular carcinoma: part II. Extracellular agents, hepatobiliary agents, and ancillary imaging features. Radiology 2014;273:30-50.

28. Muramatsu Y, Nawano S, Takayasu K, Moriyama N, Yamada T, Yamasaki S, et al. Early hepatocellular carcinoma: MR imaging. Radiology 1991;181:209-13.

29. Hussain SM, Zondervan PE, IJzermans JNM, Schalm SW, de Man RA, Krestin GP. Benign versus malignant hepatic nodules: MR imaging findings with pathologic correlation. RadioGraphics 2002;22:1023-36.

30. Shah S, Shukla A, Paunipagar B. Radiological features of hepatocellular carcinoma. J Clin Exp Hepatol 2014;4:S63-6.

31. Ebara M, Fukuda H, Kojima Y, Morimoto N, Yoshikawa M, Sugiura N, et al. Small hepatocellular carcinoma: relationship of signal intensity to histopathologic findings and metal content of the tumor and surrounding hepatic parenchyma. Radiology 1999;210:81-8 
32. Khatri G, Merrick L, Miller FH. MR imaging of hepatocellular carcinoma. Magn Reson Imaging Clin N Am 2010;18:421-50.

33. Niendorf E, Spilseth B, Wang X, Taylor A. Contrast enhanced MRI in the diagnosis of HCC. Diagnostics 2015;5:383-98.

34. Zech CJ, Reiser MF, Herrmann KA. Imaging of hepatocellular carcinoma by computed tomography and magnetic resonance imaging: state of the art. Dig Dis 2009;27:114-24.

35. Enomoto S, Tamai H, Shingaki N, Mori Y, Moribata K, Shiraki $\mathrm{T}$, et al. Assessment of hepatocellular carcinomas using conventional magnetic resonance imaging correlated with histological differentiation and a serum marker of poor prognosis. Hepatol Int 2011;5:730-7.

36. Choi JY, Lee JM, Sirlin CB. CT and MR imaging diagnosis and staging of hepatocellular carcinoma: part I. Development, growth, and spread: key pathologic and imaging aspects. Radiology 2014;272:635-54.
37. Kutami R, Nakashima $Y$, Nakashima O, Shiota K, Kojiro M. Pathomorphologic study on the mechanism of fatty change in small hepatocellular carcinoma of humans. J Hepatol 2000; 33:282-9.

38. Costa AF, Thipphavong S, Arnason T, Stueck AE, Clarke SE. Fat-containing liver lesions on imaging: detection and differential diagnosis. Am J Roentgenol 2017;210:68-77.

39. Altekruse SF, McGlynn KA, Reichman ME. Hepatocellular carcinoma incidence, mortality, and survival trends in the United States from 1975 to 2005. J Clin Oncol 2009;27:148591.

40. Zhang BH, Yang BH, Tang ZY. Randomized controlled trial of screening for hepatocellular carcinoma. J Cancer Res Clin Oncol 2004;130:417-22. 